\title{
Sinhala translation and adaptation of the Children's Somatization Inventory-24
}

ADMA Herath, KDDR Goonawardane, DP Yasira, THR Samanmalie, SDV Wikramaarachchi, RMG Karunarathne, S Rajindrajith, S Seneviratne

DOI: http://doi.org/10.4038/sljpsyc.v8i1.8134

\section{Background}

Somatic symptoms in children constitute a major burden in hospital settings and outpatient departments. Somatic symptoms are often a manifestation of internalization of symptoms in children with distress or anxiety spectrum disorders. Furthermore, since cultural background influences the ways in which a person manifests distress, the influence of cultural variability needs to be considered when interpreting somatic symptoms in children.

Aims

The objective of this study was to adapt the Children's Somatization Inventory-24 (CSI-24) for use among Sri Lankan Sinhalese speaking children.

Methods

Cultural adaptation and translation of the CSI-24 was done using the Delphi technique. After translation of the scale into Sinhalese, seven experts were chosen to rate the cultural appropriateness and content validity of each stem of the CSI 24 for use among the Sinhalese population, using a five point likert scale. After discussion and consensus, the tool was pre-tested among ten school children, prior to final evaluation.

\section{Results}

The translated tool had twenty-four items, similar to the original tool, resulting in no change in the scoring system. After discussion among the experts, certain items of the scale were modified and adapted to suit the local Sri Lankan context.

\section{Conclusions}

Through this study, we were able to develop a Sinhala translation of the CSI-24, which is culturally acceptable, and which has sound judgmental validity for the measurement of severity of somatic symptoms in Sri Lankan Sinhalese speaking children.

SL J Psychiatry 2017; 8(1): 17-20

\section{Background}

Somatic symptoms in children constitute a major burden in hospital settings and outpatient departments. Between $31 \%-49 \%$ of children between the ages of 11 to 17 years meet the criteria for somatoform disorder, and of these $7.2 \%$ fulfill the criteria for somatisation disorder according to the DSM IV classification (1-3). Even though somatic symptoms are common in children, only a minority meet the diagnostic criteria for somatisation disorder (4).

Previous authors have suggested the importance of a separate operational criteria in children for diagnosis of somatic symptoms, including the use of developmentally appropriate criteria $(4,5)$. Somatic symptoms are often a manifestation of internalisation of symptoms in children with distress or anxiety spectrum disorders (5). Furthermore, since cultural background influences the ways in which a person manifests distress, the influence of cultural variability also has to be considered when interpreting somatic symptoms in children $(5,6)$. A study conducted at the outpatient department of Lady Ridgeway Hospital for Children, Colombo, Sri Lanka reports that $56 \%$ of children missed school and in $46 \%$ daily living was significantly disrupted, due to the impact of somatic symptoms highlighting the importance and severity of this condition in Sri Lanka too (6).

These findings indicate the need for a tool to measure the severity of somatic symptoms in Sri Lankan children. To date there is no such culturally adapted tool for use in Sri Lanka. This study attempts to adapt and translate a tool for measurement of the severity of somatic symptoms in children - namely the Children's Somatization Inventory-24 (CSI-24) - which could be utilised in both clinical and community settings (6). The Children's Somatization Inventory-24 (CSI-24) is a refined version of the Children's Somatization 
Inventory-35 developed by Walker et al., in 2001 (6). The symptoms in CSI-35 were derived from the Child Behavior Check List and the DSM IV (6). This tool can be applied for children and adolescents between the ages of 8 to 18 years of age, and has been validated and translated to Chinese, Turkish and Polish languages (7, 8). It has a good internal consistency, with an alpha level of 0.87 and a correlation of 0.23 , which indicates the efficacy of this tool in differentiating somatic symptoms from the general construct of somatization $(12,13)$. Although not formally validated for use in Sri Lanka, this tool has been translated by forward and backward translation method and used in two previous local studies, which examined correlations between somatic symptoms, constipation and abuse $(15,16,17)$. In this study we have further adapted the Children's Somatization Inventory-24 (CSI-24) for use among Sri Lankan Sinhalese speaking children, in order to improve the content and face validity of this tool for use in this country.

\section{Method}

Permission for cultural adaptation and translation of the Children's Somatization Inventory-24 (CSI-24), was granted by the original author, Professor Lynn S Walker, Professor of Paediatrics, Vanderbilt University, USA. Ethical clearance for the study was obtained from the Ethical Review Committee of the Lady Ridgeway Hospital for Children.

Cultural adaptation and translation was done using the Delphi technique, as described in previous literature (18). Seven experts were chosen to rate the cultural appropriateness and content validity of each stem of the CSI 24 for use among the Sinhalese population, using a five point Likert scale. The agreeableness was measured by pre-defined criteria (Annexure 1). To ensure the comprehensiveness of the process, seven experts from different backgrounds were included in the validation process. These experts included a consultant child and adolescent psychiatrist, two senior registrars in child psychiatry, one registrar in psychiatry, a social worker, medical officer and a nursing officer (18). The experts were selected from the same unit for reasons of feasibility; since the average time for translations was about eight hours and all seven experts had to be gathered together at a single given time and venue, to carry out the study methodology.

Initially, in order to eliminate bias, each expert was asked to view the original version of the CSI 24 separately and was individually asked to rate it for appropriateness. They were also asked to translate each item individually according to the pre-defined criteria. During the translation process, each person was asked to focus more on content validity rather than semantic validity. Thereafter, the seven panelists gathered at one place to discuss and reach a consensus; the translated versions of the tool were exchanged among the seven experts. Each expert was asked to rate each of the translated versions of the tool according to predefined criteria (Annexure 1) $(19,20)$. Thus each expert received seven translations of the tools for rating purposes. These seven translations were numbered and thus anonymous. When rating scale items for the appropriateness of the content and cultural acceptance, a five point Likert scale was used from 1 to 5 , with 1 indicating not agreeable and 5 indicating highly agreeable (Annexure 1). Raters were asked to rate each item of the translated tool from 1 to 5 , based on cultural appropriateness and content validity. With regards to scoring, items that rated scores of four or more were considered to have good cultural and content appropriateness; and items that scored between 2-4 were considered to need further modifications. If any item received at least one rating of less than 2 , the item was rejected as being culturally inappropriate.

In order to achieve group consensus, more than $50 \%$ of the group had to reach agreement on each modified stem of the Sinhala version of the scale. Each item was discussed until the above consensus was reached. Group members were clearly instructed not to merely agree with others in the group, during the process of consensus generation.

Thereafter, the translated tool was administered to a group of ten children for pre-testing. Three statements were modified following the pre-testing, with amendment of certain Sinhalese terms, which children aged 8-18 years found difficult to comprehend. The final translation was reviewed by a paediatric gastroenterologist and a consultant child and adolescent psychiatrist with regards to judgemental validity. The tool was then back translated by a bilingual translator, who did not have a medical background, who was not aware of the original CSI 24.

\section{Results}

The translated tool had twenty-four items, similar to the original tool, resulting in no change in the scoring system. Therefore the method of scoring remained unchanged from that of the original CSI-24 (12).

After the first round, all seven translators showed good agreement regarding nine items out of the of 24 translated items. There was fairly good consensus agreement among the translators about six items and poor agreement regarding the remaining nine items. After discussion the seven experts slightly modified the content and terms of item numbers 3,5,7,8,11,12,13,15, and 16. Details of these modifications are listed below.

\section{Item number 3, pain in your heart or chest}

The term pain in heart was considered culturally insensitive since the Sinhalese language does not visualize the heart or chest separately. Therefore the item was modified and simplified to imply pain in chest. 
Item number 5, pain in your lower back

Since children do not often demarcate back pain to the lower back area, this statement was modified to imply pain in the back.

Items 7 and 16, trouble getting your breath when you are not exercising and your heart beating too fast even when you are not exercising.

As the concept of regular exercise was novel to Sri Lankan culture, the stem was modified to contain the word 'at rest'.

Items 8 and 12, hot or cold spells (suddenly feeling hot or cold for no reason); nausea or upset stomach (feeling like you might throw up or have an upset stomach.)

It was felt that the word spells may not be familiar to children of a Sinhalese speaking background. Therefore the word spells was removed and only the sentence in brackets was retained.

Item 11, heavy feelings in your arms or legs (when they feel too heavy to move)

This item was modified to imply the meaning that arms or legs feel too heavy to move, because the term heavy feelings was considered to be culturally inappropriate. It was discussed that Sri Lankan children might understand the direct translation of word feelings as a thought or an emotion rather than a sensation.

Item 13, constipation (when it's hard to have a B.M. or go poop.)

In this stem, the part within the bracket was removed since even though there is a commonly used term for go poop among Sinhalese children, it was considered inappropriate to be expressed in a questionnaire.

Item 15 , pain in your stomach or abdomen

In this stem, the word stomach was removed since Sri Lankan children use the word abdomen or tummy rather than stomach. The term stomach is associated with a different Sinhalese term which does not reflect pain in the abdomen. Thus the term was modified as pain in the abdomen.

\section{Discussion}

There are many ways of translating and culturally adapting tools or rating scales for use in different cultural backgrounds. The method adopted in this study is a translation process that combines qualitative and quantitative methodology (18-20). Similar methodology has been described when translating the Bradford Somatization Inventory for use among the Sinhalese population (18). Some may argue that direct translation of the original version is more appropriate since it gives a word-to-word translation of the original items. However, in the context of somatic symptoms, constructs may vary according to culture, age and gender (13). Therefore rather than direct translation, a Delphi technique was used to culturally adapt the tool before translation, since it is a widely accepted method of cultural adaptation and translation. The hundred percent agreement by all seven experts, for any one item in this process was 0.375, which is in contrast to direct translation which shows $100 \%$ agreeability with regards to each item. The Delphi technique is a widely used and accepted method of gathering data from respondents within a domain of expertise. The technique is designed as a group communication process, which aims to achieve a convergence of opinion on a specific issue. This is a well suited method for consensus building using questionnaires and multiple iterations to arrive at a consensus.

There are certain challenges to this method of cultural adaptation of rating scales. It can be a time consuming process; the average time spent on this process is about 8 hours. It is also a process where different experts meet together to arrive at a consensus, and therefore work schedules of the different experts have to be taken into account; and there need to be a clear process of communication between the experts. Despite these challenges we were able to successfully carry out the process of translation and adaptation of the CSI-24 for Sri Lankan children, using this technique.

\section{Limitations}

This study was carried out in a tertiary care setting only; terminology and culturally used terms for symptoms in Sri Lanka can vary slightly from one part of the country to another, which would not be reflected in our scale. We initially planned to include a paediatric trainee as well, for the adaptation and consensus building process. However, due to difficulties in co-ordinating suitable times for everyone, this was not possible.

\section{Conclusions}

Through this study, we were able to develop a Sinhala translation of the CSI-24, which is culturally acceptable, and which has sound judgmental validity for the measurement of severity of somatic symptoms in Sri Lankan Sinhalese speaking children. The authors plan to further assess the reliability of this tool, by exploring internal consistency and factor structure by factor analysis in future studies.

\section{Acknowledgements}

The authors would like to thank Professor Lynn Walker, Professor in Pediatrics, Vanderbilt University, who gave the permission to validate CSI-24, Mr. Jayakumar who was kind enough to back translate the final translated tool, and Mrs. Sandyanganie, nursing officer of ward 19-B, Lady Ridgeway Hospital for children. 


\section{Conflict of Interests}

None declared

ADMA Herath, KDDR Goonawardena, DP Yasira, THR Samanmalie, SDV Wikramarachchi, RMG Karunarathne, S Senavirathne, Lady Ridgeway Hospital for Children

S Rajindrajith, Faculty of Medicine, University of Kelaniya

Corresponding author: ADMA Herath

Email: anuradhaherath1981@gmail.com

http://orcid.org/0000-0002-9312-367X

\section{References}

1. Campo JV, Fritsch SL. Somatization in children and adolescents. J Am Acad Child Adolesc Psychiatry 1994; 33(9): 1223-35.

2. Janiak-Baluch B, Lehmkuhl G. Psychological disorders and somatoform symptoms in the outpatient pediatric practice. Prax Kinderpsychol Kinderpsychiatr 2013; 62(9): 654-69.

3. Andresen JM, Woolfolk RL, Allen LA, et al. Physical symptoms and psychosocial correlates of somatization in pediatric primary care. Clin Pediatr (Phila) 2011; 50(10): 904-9.

4. Rask CU, QrnbQl E, Fink PK, Skovgaard AM. Functional somatic symptoms and consultation patterns in 5- to 7-yearolds. Pediatrics 2013 ; 132(2): 459-67.

5. American Psychiatric Association (APA). Diagnostic and Statistical Manual of Mental Disorders. 5th ed. APA; Washington, DC; 2013.

6. Perera H, Asiri ASD. Medically unexplained physical symptoms in children in a non-specialist paediatric setting. Sri Lanka J Child Health 2007; 36: 102-6.

7. Walker LS, Garber J, Green JW. Somatization symptoms in pediatric abdominal pain patients: relation to chronicity of abdominal pain and parent somatization. J Abnorm Child Psychol 1991; 19(4): 379-94.

8. Derogatis LR, Lipman RS, Rickels K, Uhlenhuth EH, Covi L. The Hopkins Symptom Checklist (HSCL): a self-report symptom inventory. Behav Sci 1974; 19(1): 1-15.

9. Walker LS, Williams SE, Smith CA, Garber J, Van Slyke DA, Lipani TA. Parent attention versus distraction: impact on symptom complaints by children with and without chronic functional abdominal pain. Pain 2006; 122(1-2): 43-52.

10. Garber J, Walker LS, Zeman J. Somatization symptoms in a community sample of children and adolescents: further validation of the Children's Somatization Inventory. Psychol Assess 1991; 3(4): 588-95.

11. Litcher L, Bromet E, Carlson G, et al. Ukrainian application of the Children's Somatization Inventory: psychometric properties and associations with internalizing symptoms. J Abnorm Child Psychol 2001; 29(2): 165-75.

12. Meesters C, Muris P, Ghys A, Reumerman T, Rooijmans M. The Children’s Somatization Inventory: further evidence for its reliability and validity in a pediatric and community sample of Dutch children and adolescents. J Pediatr Psychol 2003; 28(6): 413-22.

13. Walker LS, Beck JE, Garber J, Lambert W. Children's Somatization Inventory: psychometric properties of the revised form (CSI-24). J Paediatr Psychol 2009; 34(4): 430-40.

14. Garber J, Walker LS, Zeman J. somatization symptoms in a community sample of children and adolescents; further validation of children's somatization inventory; psychological assessment; a jounrnal of consulting and clinical psychology 1991; 4: 588-95.

15. Cecilia A. Essau BO, Bokszczanin A, Gilvarry C, Bray D. Somatic symptoms among children and adolescents in Poland: a confirmatory factor analytic study of the Children Somatization Inventory. Front Public Health 2013; 1: 72.

16. Rajiindrajit S, Devanarayana NM, Weerasooriya L, Hathagoda W, Benninga M. Quality of life and somatic symptoms in children with constipation: a school based study. J Paediatr 2013; 163(4): 1069-72.

16. Devanarayana NM, rajindrajith S, Perera M, Nishanthanie S, Karunanayake A, Benninga M. Association between functional gastrointestinal diseases and exposure to abuse in teenagers. J Trop Paediatr 2014; 60(5): 386-92.

17. Rajindrajith S, Devanarayana NM, Lakmini C, Subasinghe V, De Silva H, Benninga M. Association between child maltreatment and constipation: a school base survey using Rome 111 criteria. J Paediatr Gastroenterol Nutr 2014; 58(4): 486-90.

18. Sumathipala A, Murray J. New approach to translating instruments for cross-cultural research: a combined qualitative and quantitative approach for translation and consensus generation. Int J Methods Psychiatr Res 2000; 9(2): 87-95.

19. Jones J, Hunter D. Consensus methods for medical and health services research. BMJ 1995; 311(7001): 376-80.

20. Scot EA, Black NA. When does consensus exist in expert panels? J Public Health Med 1991; 13(1); 35-9.

\begin{tabular}{|c|c|}
\hline Likert scale score & Degree of agreeableness \\
\hline 5 & $\begin{array}{l}\text { Fully agree on the meaning } \\
\text { given by translation }\end{array}$ \\
\hline 4 & $\begin{array}{l}\text { Fairly satisfactory meaning, } \\
\text { may need word changes }\end{array}$ \\
\hline 3 & $\begin{array}{l}\text { Not so satisfactory meaning } \\
\text { with some word changes } \\
\text { definitely needed }\end{array}$ \\
\hline 2 & $\begin{array}{l}\text { Not satisfactory meaning but } \\
\text { some words are useful }\end{array}$ \\
\hline 1 & $\begin{array}{l}\text { Totally disagree on the meaning } \\
\text { given by the translation }\end{array}$ \\
\hline
\end{tabular}

